\title{
MISSING LINKS OF miRNA REGULATION AND TRAIL;
}

Missing Links Of miRNA regulation and TRAIL mediated signaling in endometrial cancer:

do we have enough understanding

Ammad Ahmad Farooqi, Imrana Iftikhar Awan

Article Citation

Farooqi AA, Awan II. Missing Links of mirNA Regulation and Trail; missing links of mirNA regulation and trail mediated signaling in endometrial cancer: do we have enough understanding. Professional Med J 2013;20(5): 642-643.

Endometrial cancer is multifactorial disease. Genetic and mechanistic diversity creates challenges for therapy. Interestingly, high-throughput technologies, including the analyses of apoptotic pathway have considerably enhanced our current understanding of dysregulated protein network in endometrial cancer.

It is now clear that cancer proteome is exceptionally multifaceted because of regulation of gene network by downstream effectors of signaling cascades. Apoptotic response in endometrial cancer cells is impaired because of interconnectivity of proteins into complexes and signaling networks that are highly divergent in time and space.

Insights from TRAIL mediated signaling research are catalyzing new lines of study that could not only explain molecular mechanisms of disease but also highlight opportunities for therapeutic interventions.

Following features are highlighted;

- $\quad$ miRNA subsets are regulated by downstream effectors of signaling cascades.

- miRNAs regulate tumor suppressor and oncogenes in endometrial cancer cells.

- Various approaches which show promise in restoring apoptosis in endometrial cancer cells.

Increasingly it is being realized that main regulators of microRNAs biogenesis are misrepresented in endometrial cancer. These are accountable for altered microRNAome in endometrial cancer cells. Transiently transfecting miR-34b in endometrial cancer cells resulted in inhibition of cell growth, migration and most notably invasion. miR-200c negatively regulated BRD7 and facilitated entry of $\beta$-catenin in the nucleus to stimulate the expression of cyclinD1 and c-myc ${ }^{1}$.

Emerging evidence suggests that miRNA expression is also regulated by progesterone. In line with this approach, a recent study indicated that cells treated with medroxyprogesterone acetate had upregulated miR-625*, $-21,-142-5 p$, and $146 b-5 p$ by more than $400 \%$, whereas miR-633, $-29 c,-29 *$, and $-193 b$ were decreased by $50 \%{ }^{2}$.

Targeted inhibition of metadherin (MTDH) in endometrial cancer cells resulted in an increase of sensitivity of cancer cells to TRAIL-induced apoptosis $^{3}$. Similarly, Kinase suppressor of Ras 1 (KSR1), death-associated protein kinase (DAPK) and casein kinase (CK2) abrogation sensitized resistant endometrial cell lines to both TRAIL- and Fas-induced apoptosis $^{4,5,6}$.

DAPK silencing notably increased the secretion of TRAIL protein from the cells ${ }^{7}$. The multikinase inhibitor Sorafenib sensitized endometrial cancer cells to TRAIL by inhibiting FLICE-Inhibitory Protein (FLIP) ${ }^{8}$. It has been convincingly revealed that $\mathrm{DcR} 1$ expression occured in a subset of EC and contributed to resistance to TRAlL-induced apoptosis ${ }^{9}$.

It has been convincingly revealed that estrogen metabolite 2-methoxyoestradiol alone or in combination with TRAIL mediates apoptosis in cancerous cells ${ }^{10}$. 
We do not have well developed understanding of different tumor suppressors, oncogenes, activating mutations and loss of function of genes of endometrial cancer patients of our local population moreover valid predictive biomarkers for stratification of therapy are not available at present. Future studies should converge on dismantling disease associated genes and post-transcriptional processing of the genes. Without detailed and comprehensive knowledge of endometrial cancer biology and genes that predispose individuals to cancer identification of high-risk population will be difficult.

Copyright@ 05 July, 2013.

\section{REFERENCES}

1. Bae J, Won M, Kim DY, Kim JH, Kim YM, Kim YT, Nam $\mathrm{JH}$, Suh DS. Identification of differentially expressed microRNAs in endometrial cancer cells after progesterone treatment. Int J Gynecol Cancer. 2012 May;22(4):561-5.

2. Bai T, Tanaka T, Yukawa K. Targeted knockdown of death-associated protein kinase expression induces TRAIL-mediated apoptosis in human endometrial adenocarcinoma cells. Int J Oncol. 2010 Jul;37(1):203-10.

3. Kato S, Sadarangani A, Lange S, Villalón M, Bra es J, Brosens JJ, Owen Gl, Cuello M. The oestrogen metabolite 2-methoxyoestradiol alone or in combination with tumour necrosis factor-related apoptosis-inducing ligand mediates apoptosis in cancerous but not healthy cells of the human endometrium. Endocr Relat Cancer. 2007 Jun;14(2):351-68.

4. Llobet D, Eritja N, Domingo M, Bergada L, Mirantes C, Santacana M, Pallares J, Maci A, Yeramian A, Encinas M, Moreno-Bueno G, Palacios J, Lewis RE, Matias-

AUTHOR(S):

1. AMMAD AHMAD FAROOQI

Laboratory for Translational Oncology and

Personalized Medicine,

Rashid Latif Medical College,

$35 \mathrm{Km}$ Ferozepur Road, Lahore, Pakistan.

2. IMRANA IFTIKHAR AWAN

Department of Obstetrics \& Gyanecology,

Rashid Latif Medical College,

35 Km Ferozepur Road, Lahore, Pakistan.
Guiu X, Dolcet X. KSR1 is overexpressed in endometrial carcinoma and regulates proliferation and TRAIL-induced apoptosis by modulating FLIP levels. Am J Pathol. 2011 Apr;178(4):1529-43.

5. Llobet D, Eritja N, Encinas M, Llecha N, Yeramian A, Pallares J, Sorolla A, Gonzalez-Tallada FJ, Matias-Guiu $X$, Dolcet $X$. CK2 controls TRAIL and Fas sensitivity by regulating FLIP levels in endometrial carcinoma cells. Oncogene. 2008 Apr 17;27(18):2513-24.

6. Llobet D, Eritja N, Yeramian A, Pallares J, Sorolla A, Domingo M, Santacana M, Gonzalez-Tallada FJ, Matias-Guiu X, Dolcet $X$. The multikinase inhibitor Sorafenib induces apoptosis and sensitises endometrial cancer cells to TRAIL by different mechanisms. Eur J Cancer. 2010 Mar;46(4):836-50.

7. Meng X, Brachova P, Yang S, Xiong Z, Zhang Y, Thiel KW, Leslie KK. Knockdown of MTDH sensitizes endometrial cancer cells to cell death induction by death receptor ligand TRAIL and HDAC inhibitor LBH589 co-treatment. PLoS One. 2011;6(6):e20920. doi: 10.1371/journal.pone.0020920.

8. Park YA, Lee JW, Choi JJ, Jeon HK, Cho Y, Choi C, Kim TJ, Lee NW, Kim BG, Bae DS. The interactions between MicroRNA-200C and BRD7 in endometrial carcinoma. Gynecol Oncol. 2012 Jan;124(1):125-33.

9. Tanaka T, Bai T, Yukawa K. Suppressed protein expression of the death-associated protein kinase enhances 5-fluorouracil-sensitivity but not etoposide-sensitivity in human endometrial adenocarcinoma cells. Oncol Rep. 2010 Nov;24(5):1401-5.

10. Tarragona J, Llecha N, Santacana M, Lopez S, Gatius S, Llobet D, Dolcet X, Palomar-Asenjo V, Gonzalez-Tallada FJ, Matias-Guiu X. DcR1 expression in endometrial carcinomas. Virchows Arch. 2010 Jan;456(1):39-44.

Correspondence address:

Ammad Ahmad Farooqi

Laboratory for Translational Oncology and Personalized Medicine, Rashid Latif Medical College, $35 \mathrm{Km}$ Ferozepur Road, Lahore, Pakistan. ammadahmad638@yahoo.com 